\title{
Carcinosarcomas of the Uterus: Prognostic Factors and Impact of Adjuvant Treatment
}

\author{
Kerri Beckmann (D) \\ Sudarshan Selva-Nayagam ${ }^{2}$ \\ Ian Olver ${ }^{3}$ \\ Caroline Miller (iD ${ }^{4,5}$ \\ Elizabeth S Buckley iD \\ Kate Powell ${ }^{4}$ \\ Dianne Buranyi-Trevarton ${ }^{6}$ \\ Raghu Gowda ${ }^{7}$ \\ David Roder' \\ Martin K Oehler ${ }^{8}$ \\ 'Cancer Epidemiology and Population \\ Health Research, University of South \\ Australia, Adelaide, Australia; ${ }^{2}$ Medical \\ Oncology, Royal Adelaide Hospital \\ Cancer Centre, Adelaide, Australia; \\ ${ }^{3}$ Faculty of Health and Medical Sciences, \\ University of Adelaide, Adelaide, \\ Australia; ${ }^{4}$ South Australian Health and \\ Medical Research Institute, Adelaide, \\ Australia; ${ }^{5}$ School of Public Health, \\ University of Adelaide, Adelaide, \\ Australia; ${ }^{6}$ Central Adelaide Local Health \\ Network, SA Health, Adelaide, Australia; \\ ${ }^{7}$ Radiation Oncology, Royal Adelaide \\ Hospital, Adelaide, Australia; \\ ${ }^{8}$ Gynaecological Oncology, Royal \\ Adelaide Hospital, Adelaide, Australia
}

Correspondence: Kerri Beckmann

Cancer Epidemiology and Population Health Research, University of South Australia, Level 8 SAHMRI Building, PO Box 247 I North Terrace, Adelaide, South Australia, Australia

Tel +6I 883027018

Email kerri.beckmann@unisa.edu.au
Background: Uncertainties remain about the most effective treatment for uterine carcinosarcoma (UCS), a rare but aggressive uterine cancer, due to the limited scope for randomized trials. This study investigates whether nodal excision or adjuvant therapies after hysterectomy offer a survival benefit, using multi-institutional clinical registry data from South Australia.

Methods: Data for all consecutive cases of UCS from 1980 to 2019 were extracted from the Clinical Cancer Registry. Clinical and treatment-related factors associated with diseasespecific mortality (DSM) and all-cause mortality (ACM) were determined using multivariable Cox proportional hazards regression, with subgroup analyses by stage.

Results: Median follow-up for the 140 eligible cases was 21 months. 94\% underwent hysterectomy, and $72 \%$ had an additional pelvic lymph node dissection (PLND). Furthermore, 16\% received adjuvant chemotherapy; $11 \%$ adjuvant radiotherapy and $16 \%$ multimodal chemoradiotherapy, with an increase in the latter two modalities over time. DSM was reduced among those who underwent PLND (HR: 0.41; 95\%CI: 0.23-0.74), adjuvant chemotherapy (HR: 0.39; 95\%CI: $0.18-0.84$ ) or multimodality treatment (HR: 0.11 ; 95\%CI: $0.06-0.30$ ) compared with hysterectomy alone for the whole cohort and for late stage disease (FIGO III/IV) but not for earlier stage disease, except for reduced DSM with multimodal therapy. Findings were similar for ACM. Conclusion: Our findings indicate better survival among those who received PLND, chemotherapy and multimodal adjuvant therapy, with the latter applying to early and late stage disease. However, cautious interpretation is warranted, due to potential "indication bias" and limited power. Further research into effective treatment modalities, ideally using prospective study designs, is needed.

Keywords: uterine carcinosarcoma, management, survival, multimodal therapy, adjuvant chemotherapy, adjuvant radiotherapy

\section{Plain Language Summary}

Uncertainties remain about the most effective treatment for uterine carcinosarcoma, a rare but aggressive form of uterine cancer. This study used clinical data from several treatment centers to investigate whether removing lymph nodes or giving adjuvant therapies after hysterectomy offered any survival benefit. Findings indicated improved survival overall among those who had lymph nodes removes and among those who received either chemotherapy or radiotherapy plus chemotherapy after surgery. Combined adjuvant therapy improved survival in both early and late stage of disease. Some caution in interpreting these results is warranted due to the small number of women in the study and the potential to select healthier women for combined therapy.

\section{Introduction}

Uterine carcinosarcomas (UCS), previously termed malignant mixed Mullerian tumors $(\mathrm{MMMT})$, are a rare subtype $(\sim 5 \%)$ of uterine cancer. ${ }^{1}$ Their histological 
diagnosis is based on mixed epithelial and mesenchymal cell types within the tumor. ${ }^{2}$ Traditionally thought of as a class of sarcoma, molecular profiling and identification of similar risk profiles to endometrial carcinomas has led to the reclassification of UCS as carcinomas. ${ }^{3,4}$ Known to be extremely aggressive tumors, patients often present with extrauterine disease and/or distant metastases, have high rates of recurrence and relatively short survival. ${ }^{5}$ With five-year survival in the order of $30-39 \%$, outcomes are comparatively worse than those for both grade 3 endometrial cancers and uterine sarcomas. ${ }^{1}$

Surgery, including hysterectomy bilateral salpingooophorectomy and pelvic lymph node dissection is the primary treatment for UCS. Complete cytoreduction should be achieved, as this may be associated with an overall survival benefit. The therapeutic value of the lymph node removal and specific adjuvant therapies is not well known. ${ }^{6,7}$ Most studies of lymphadenectomy have shown a survival benefit, though some have suggested that there may be a threshold required in terms of number of nodes excised. ${ }^{7,8}$ Nevertheless, lymphadenectomy is recommended in corpus-confined disease to improve accuracy of disease staging and subsequent treatment planning. Given the high recurrence rate for UCS, even for early stage disease, postoperative adjuvant therapy is also recommended for all stages of disease. However, the optimal adjuvant treatment modality (ie, chemotherapy, radiotherapy (vaginal vault brachytherapy or external beam pelvic), or multimodal therapy combining chemotherapy and radiotherapy) is yet to be established and no stage-specific guidelines exist due to lack of data on efficacy. ${ }^{9}$ Survival benefits have been demonstrated with the use of adjuvant chemotherapy in advanced UCS (stages III and IV). Furthermore, results from two randomized trials have indicated that a combination of chemotherapy agents is more effective than a single chemotherapeutic agent. ${ }^{10,11}$ Common chemotherapies included ifosfamide-based regimes but more recently platinum-taxane combinations, with the latter being preferred due to lower overall toxicity. ${ }^{12,13}$ However, direct evidence for superiority of specific regimes is lacking. ${ }^{14}$ Whether adjuvant radiotherapy offers any benefit in terms of disease specific or overall survival is the subject of ongoing debate. ${ }^{9,15}$ Both vaginal brachytherapy or whole pelvic external beam radiotherapy (EBRT) are common treatment options. But while several retrospective studies have shown reduced risk of locoregional recurrence, few have demonstrated improved survival with adjuvant radiotherapy. ${ }^{5}$ Multimodal therapies incorporating adjuvant chemotherapy and radiotherapy after hysterectomy are increasingly being advocated for the management of UCS with encouraging results, ${ }^{7,9,16}$ though definitive evidence is still not available.

Given the rarity of this tumors type, testing the efficacy of different treatment modalities via clinical trials has proven difficult and considerable uncertainty remains around optimal treatment. There is, therefore, a continuing need to investigate treatment patterns and outcomes though retrospective analyses of cohorts of UCS accrued over time. The objective of this study was to describe the clinical characteristics, treatment patterns including use of adjuvant therapies, and survival outcomes in a multi-institutional cohort of women with UCS managed within the public tertiary setting in Adelaide, Australia. We specifically address whether nodal excision and or adjuvant therapy in addition to hysterectomy offer any survival benefit in women with UCS.

\section{Methods}

The study cohort consisted of consecutive cases of UCS reported within the South Australian Clinical Cancer Registry (SACCR). A multi-institutional registry, the SACCR was established in the 1980s to monitor the clinical characteristics, primary treatments and outcomes for patients admitted to the major public teaching hospitals in Adelaide, South Australia. Clinical data were collected through manual review of patient medical records including histopathology reports, and more recently through electronic patient record review. The study cohort included all women who were diagnosed with carcinosarcoma of the uterus between 1980 and 2019 and were resident in South Australia. All identified cases were reviewed to ensure histopathology reporting confirmed classification as UCS. Details were extracted from the SACCR for demographic characteristics (eg, age at diagnosis, year of diagnosis, country of birth and residential postcode); clinical characteristics (eg, stage based on the International Federation of Gynaecology and Obstetrics (FIGO) criteria, grade classified as poorly, moderately or well differentiated, heterologous-homologous classification based on sarcomatous component, myometrial invasion $(<50 \%$ or $\geq 50 \%$ ), lymphovascular invasion, and nodal involvement); primary treatment types and start dates (for hysterectomy, nodal excision, radiotherapy - external beam and/or brachytherapy, hormonal therapy, chemotherapy regimen); and date and cause of death (uterine cancer, other cancer 
or other cause). Area level measures of socioeconomic status were assigned according to the Australian Bureau of Statistic's Socioeconomic Indexes for Area (SEIFA) deprivation scores ${ }^{17}$ for residential postcodes, categorized into quartiles from most to least deprivation for the South Australian population. Place of residence was classified as metropolitan or other (inner regional/rural/remote/very remote) according to the Accessibility/Remoteness Index of Australia, ${ }^{18}$ based on residential postcodes. Country of birth was classified as Australia, other English-speaking country, other non-English speaking country or unknown. Date and cause of death were determined through linkage with the states' central cancer registry which receives regular updates from the State Register of Births, Deaths and Marriages, and periodically from the National Death Index. Since the focus of data collection in SACCR relates to primary treatment, data on disease recurrence and subsequent treatment were incomplete and not included in this analysis.

Categorical variables for demographic and clinical characteristics and primary treatments are presented as frequencies and percentages. Differences in receipt of specific treatments were compared via chi-squared tests. Factors associated with giving adjuvant therapy (any radiotherapy, chemotherapy or multimodal radiochemotherapy following hysterectomy) were assessed using multivariable binary logistic regression. Diseasespecific survival (DSS) and overall survival (OS) were assessed using Kaplan-Meier methods, and differences in crude survival tested via log rank tests. Survival times were calculated from date of diagnosis to date of death with censoring at last recorded date of follow-up (up to March 2020) if not deceased. Factors associated with time to disease specific mortality (DSM) and for all-cause mortality (ACM) were assessed using multivariable Cox proportional hazards regression models. Proportional hazards assumptions were tested using Schoenfeld residuals test. Subgroup analyses were also undertaken to assess the association of different treatment approaches with risk of death in women with early (FIGO stage I/II) and advanced (FIGO stage III/IV) disease. An additional subgroup analysis was performed for the earliest carcinosarcoma stage alone (FIGO stage 1), given the uncertainties about indications and benefits of adjuvant treatment in this group of patients. ${ }^{19}$ All statistical tests were two-sided and $p$-values $<0.05$ were accepted to show statistically significant associations.
This study was conducted in accordance with the Declaration of Helsinki and received ethical approval from the SA Department for Health and Ageing Human Research Ethics Committee (HREC/17/SAH/69) with a waiver of consent for use of deidentified data from the clinical registry.

\section{Results}

We identified 149 women with UCS who were managed within public teaching hospitals in South Australia. Nine women were excluded because they were resident in a different state or had only received their diagnosis at one of the included hospitals. Sociodemographic and clinical characteristics for the study cohort are presented in Table 1. Age at diagnosis ranged from 41-95 years, with a median age of 71 years. Other sociodemographic characteristics were reflective of broader distribution within South Australia. Almost half of the cohort had early stage disease at diagnosis (FIGO I/II) and the other half advanced stage (FIGO III/IV) disease (48\% and 51\%, respectively). Seventy-seven percent of cases involve myometrial involvement and 15\% lymph node metastasis. Fifteen percent of women presented with distant metastasis at diagnosis. Fifty percent of the UCS had a major sarcomatous component, with $35 \%$ classified as heterologous.

Treatment patterns are shown in Table 2. The majority of women were treated with curative intent. Almost all (94\%) received surgery, most commonly an extended hysterectomy. Nodal excision was performed in $72 \%$ of women. A total of $44 \%$ of women received adjuvant therapy, most frequently adjuvant chemotherapy $(16 \%)$ or multimodal adjuvant therapy (16\%). Eleven percent received adjuvant radiotherapy only. Chemotherapy regimens most frequently consisted of combinations of taxane and platinum-based agents (carboplatin + paclitaxel being the most common (49\%)), though combinations including cyclophosphamide and doxorubicin were also frequently given, particularly in earlier periods. Radiotherapy was delivered either via EBRT (38\%), vaginal brachytherapy $(18 \%)$ or via a combination of brachytherapy and EBRT (18\%), with radiotherapy type not being reported in $26 \%$ of cases. 3D conformal radiation treatment (3DCRT) was used between 2005 and 2014 and intensity modulated radiation therapy (IMRT) since 2015. Total dose during both the 3DCRT and IMRT era was $45 \mathrm{~Gy}$ at $1.8 \mathrm{~Gy} / \mathrm{F}$. For vaginal brachytherapy, total dose was $21 \mathrm{~Gy} / 3 \mathrm{~F}$ over one week if used alone or $11 \mathrm{~Gy} / 2 \mathrm{~F}$ if used following EBRT. 
Table I Demographic and Clinicopathological Characteristics of UCS Cases

\begin{tabular}{|c|c|c|c|}
\hline \multicolumn{2}{|c|}{ Demographic and Clinical Characteristics } & \multirow{2}{*}{$\frac{n}{140}$} & \multirow{2}{*}{$\frac{\%}{100}$} \\
\hline Total & & & \\
\hline \multirow[t]{8}{*}{ Age group (years) } & $<50$ & 4 & 3 \\
\hline & $50-54$ & 8 & 6 \\
\hline & $55-59$ & 6 & 4 \\
\hline & $60-64$ & 14 & 10 \\
\hline & $65-69$ & 29 & 21 \\
\hline & $70-74$ & 29 & 21 \\
\hline & $75-79$ & 21 & 15 \\
\hline & $80+$ & 29 & 21 \\
\hline \multirow[t]{7}{*}{ Period of diagnosis } & $<1990$ & 24 & 17 \\
\hline & 1990-94 & 20 & 14 \\
\hline & $1995-99$ & 22 & 16 \\
\hline & $2000-04$ & 12 & 9 \\
\hline & $2005-09$ & 23 & 16 \\
\hline & $2010-14$ & 20 & 14 \\
\hline & $2015-19$ & 19 & 14 \\
\hline \multirow[t]{2}{*}{ Residence } & Metropolitan & 111 & 79 \\
\hline & Outer urban/rural/remote & 29 & 21 \\
\hline \multirow{5}{*}{$\begin{array}{l}\text { Socioeconomic status } \\
\text { (SEIFA SA quartiles) }\end{array}$} & Most disadvantaged & 44 & 31 \\
\hline & Lower middle & 31 & 22 \\
\hline & Upper middle & 30 & 21 \\
\hline & Least disadvantaged & 35 & 25 \\
\hline & Unknown & 9 & 5 \\
\hline \multirow[t]{3}{*}{ Country of birth } & Australia & 90 & 64 \\
\hline & $\begin{array}{l}\text { English speaking (UK/Ireland/ } \\
\text { NZ) }\end{array}$ & 26 & 19 \\
\hline & Other & 24 & 17 \\
\hline \multirow[t]{5}{*}{ FIGO stage } & I & 58 & 41 \\
\hline & II & 10 & 7 \\
\hline & III & 42 & 30 \\
\hline & IV & 29 & 21 \\
\hline & Not reported & 1 & I \\
\hline
\end{tabular}

(Continued)
Table I (Continued).

\begin{tabular}{|c|c|c|c|}
\hline \multicolumn{2}{|c|}{ Demographic and Clinical Characteristics } & \multirow{2}{*}{$\begin{array}{l}\mathbf{n} \\
5\end{array}$} & \multirow{2}{*}{$\begin{array}{l}\% \\
4\end{array}$} \\
\hline Grade & Well differentiated & & \\
\hline & Moderately differentiated & 15 & $\mathrm{II}$ \\
\hline & High/undifferentiated & 95 & 68 \\
\hline & Unknown & 25 & 19 \\
\hline \multirow{3}{*}{$\begin{array}{l}\text { Sarcomatous } \\
\text { component }\end{array}$} & Minor & 50 & 36 \\
\hline & Major & 70 & 50 \\
\hline & Unknown & 20 & 14 \\
\hline \multirow[t]{3}{*}{ Classification } & Homologous & 75 & 53 \\
\hline & Heterologous & 49 & 35 \\
\hline & Unknown & 16 & II \\
\hline \multirow{3}{*}{$\begin{array}{l}\text { Lymphovascular } \\
\text { invasion }\end{array}$} & No & 40 & 29 \\
\hline & Yes & 54 & 39 \\
\hline & Unknown & 46 & 33 \\
\hline \multirow{7}{*}{$\begin{array}{l}\text { Lymph node } \\
\text { involvement }\end{array}$} & No & 50 & 36 \\
\hline & Yes & 18 & 15 \\
\hline & $\mathrm{I}$ & 5 & 4 \\
\hline & 2 & 3 & 2 \\
\hline & $3+$ & 10 & 7 \\
\hline & Unknown & 44 & 31 \\
\hline & Not examined & 28 & 20 \\
\hline \multirow{3}{*}{$\begin{array}{l}\text { Myometrial } \\
\text { involvement }\end{array}$} & No & 7 & 5 \\
\hline & Yes & 108 & 77 \\
\hline & Unknown & 25 & 18 \\
\hline \multirow{3}{*}{$\begin{array}{l}\text { Parametrial } \\
\text { involvement }\end{array}$} & No & 81 & 57 \\
\hline & Yes & 33 & 24 \\
\hline & Unknown & 26 & 19 \\
\hline \multirow[t]{3}{*}{ Adnexal involvement } & No & 87 & 62 \\
\hline & Yes & 29 & 21 \\
\hline & Unknown & 24 & 17 \\
\hline
\end{tabular}


Table I (Continued).

\begin{tabular}{|l|l|c|c|}
\hline Demographic and Clinical Characteristics & $\mathbf{n}$ & $\%$ \\
\hline Omental involvement & No & 59 & 42 \\
\cline { 2 - 4 } & Yes & 24 & 17 \\
\cline { 2 - 4 } & Unknown & 57 & 41 \\
\hline \multirow{3}{*}{ Distant metastases } & No & 118 & 84 \\
\cline { 2 - 5 } & Yes & 21 & 15 \\
\cline { 2 - 5 } & Unknown & 1 & 1 \\
\hline
\end{tabular}

Overall, $36 \%$ of the cohort experienced recurrence while $28 \%$ were never disease free. Recurrence was most likely to involve distant metastases. (Table 2)

Results of multivariable analysis for receipt of any form of adjuvant therapy are presented in Table 3 . Factors most strongly associated with adjuvant therapy were age, period of diagnosis and FIGO stage. Compared with women $<60$ years, those $\geq 70$ years were much less likely to receive adjuvant therapy (Odds ratio, OR: 0.10 ; 95\%CI: 0.02-0.62), while those with stage III compared with stage I disease were much more likely to receive adjuvant therapy (OR: 10.8; 95\%CI: 3.22-49.7). Adjuvant therapy was offered less frequently during the 1990s compared with the 1980s (OR: 0.14; 95\%CI: 0.020.68-49.7) but has since increased to similar levels as in the 1980s. No other clinicopathological characteristics were found to be associated with having adjuvant therapy.

Clinicopathological factors associated with receiving radiotherapy, chemotherapy or multimodal adjuvant therapy (separately) are shown in Supplementary Tables 1 and 2. Use of adjuvant radiotherapy has increased over time, while use of chemotherapy initially decreases before returning to similar levels as during the 1980s. Receipt of radiotherapy was not associated with stage at diagnosis. However, receipt of chemotherapy was strongly associated later stage disease (OR: 11.3; 95\% CI: 2.62-48.8 for FIGO III/IV vs I-II). The only factors found to be associated with likelihood of receiving multimodal adjuvant therapy were age at diagnosis (OR: 0.87; 95\%CI: 0.81-0.95) and year of diagnosis (OR: 1.16; 95\%CI: 1.05-1.29).

Median follow-up for the cohort overall was 21 months. Median time to death was 13 months for uterine
Table 2 Treatment Characteristic for Women with UCS

\begin{tabular}{|c|c|c|c|}
\hline \multicolumn{2}{|c|}{ Primary Treatment Characteristics $(n=140)$} & \multirow{2}{*}{$\frac{\mathbf{n}}{3}$} & \multirow[t]{2}{*}{$\%$} \\
\hline Treatment intent & No treatment & & \\
\hline & Curative & 74 & 53 \\
\hline & Control/life prolonging & 21 & 15 \\
\hline & Palliative & 34 & 24 \\
\hline & Unknown & 8 & 5 \\
\hline \multirow{4}{*}{$\begin{array}{l}\text { Treatment } \\
\text { approach }\end{array}$} & No surgery & 8 & 5 \\
\hline & $\begin{array}{l}\text { Surgery (radical/extended } \\
\text { hysterectomy) }\end{array}$ & 132 & 95 \\
\hline & Surgery alone & 70 & 50 \\
\hline & $\begin{array}{l}\text { Surgery plus adjuvant chemo- and/ } \\
\text { or radiotherapy }\end{array}$ & 62 & 44 \\
\hline \multirow{7}{*}{$\begin{array}{l}\text { Primary } \\
\text { treatments }\end{array}$} & No primary treatment & 7 & 6 \\
\hline & Hormone therapy alone & I & I \\
\hline & Surgery (any type) alone & 63 & 45 \\
\hline & Surgery + hormone therapy & 7 & 5 \\
\hline & Surgery + chemotherapy & 23 & 16 \\
\hline & Surgery + radiotherapy & 16 & 11 \\
\hline & $\begin{array}{l}\text { Surgery }+ \text { chemotherapy }+ \\
\text { radiotherapy }\end{array}$ & 23 & 16 \\
\hline \multirow[t]{3}{*}{ Nodes removed } & No & 22 & 16 \\
\hline & Yes & 102 & 73 \\
\hline & Unknown & 16 & 11 \\
\hline \multirow[t]{4}{*}{ Margin status } & No residual tumors & 68 & 49 \\
\hline & Residual tumors & 35 & 25 \\
\hline & $\begin{array}{l}\text { Margins not evaluated/not } \\
\text { reported }\end{array}$ & 29 & 21 \\
\hline & No primary site surgery & 8 & 6 \\
\hline \multirow{4}{*}{$\begin{array}{l}\text { Radiotherapy type } \\
(n=39)\end{array}$} & External beam & 15 & 38 \\
\hline & Brachytherapy & 7 & 18 \\
\hline & $\begin{array}{l}\text { External beam and brachytherapy } \\
\text { combined }\end{array}$ & 7 & 18 \\
\hline & Unknown & 10 & 26 \\
\hline $\begin{array}{l}\text { Chemotherapy } \\
\text { type }(n=46)\end{array}$ & Cyclophosphamide (single agent) & I & I \\
\hline
\end{tabular}

(Continued) 
Table 2 (Continued).

\begin{tabular}{|c|c|c|c|}
\hline \multicolumn{2}{|c|}{ Primary Treatment Characteristics $(n=140)$} & \multirow{2}{*}{$\frac{\mathbf{n}}{4}$} & \multirow[t]{2}{*}{$\%$} \\
\hline & Doxorubicin (single agent) & & \\
\hline & Carboplatin (single agent) & I & 1 \\
\hline & Cyclophosphamide + doxorubicin & 5 & II \\
\hline & $\begin{array}{l}\text { Cyclophosphamide }+ \text { chlorambucil } \\
+ \text { doxorubicin }\end{array}$ & I & 1 \\
\hline & $\begin{array}{l}\text { Cisplatin }+ \text { cyclophosphamide }+ \\
\text { doxorubicin }\end{array}$ & 3 & 7 \\
\hline & Cisplatin + doxorubicin & 5 & 11 \\
\hline & Carboplatin + paclitaxel & 22 & 49 \\
\hline & Carboplatin + doxorubicin & I & 1 \\
\hline & Unknown (single agent) & 3 & 7 \\
\hline \multirow[t]{5}{*}{ Failure pattern } & Never disease free & 39 & 28 \\
\hline & No recurrence reported & 50 & 36 \\
\hline & Regional recurrence & 11 & 8 \\
\hline & Distant recurrence & 37 & 26 \\
\hline & Recurrence, unknown site & 3 & 2 \\
\hline
\end{tabular}

malignancy and 18 months for death from any cause. Disease specific survival at one-, two- and five-years postdiagnosis were $68 \%, 49 \%$ and $35 \%$ respectively (Table 4 ). Significant differences in crude DSS were observed by age group (log-rank $p=0.024)$, FIGO stage $(p<0.001)$, whether lymph nodes were excised or not $(p=0.003)$ and treatment modality $(p<0.001)$, with the best DSS outcomes for women who underwent multimodal therapy (surgery, adjuvant radiotherapy and adjuvant chemotherapy). Similar differences were observed for overall survival (not shown). Figure 1 presents Kaplan-Meier curves for overall survival according to treatment modality, indicating better survival among those who received multimodal therapy, especially among those with later stage disease.

Results of multivariable Cox regression models confirmed findings from crude analyses with respect to treatment approaches. With regard to DSM (Table 5), risk of death increased with increasing FIGO stage (eg, stage III vs I: HR: 3.40: 95\%CI: 1.73-6.69) and was approximately 50\% lower among women who had any lymph nodes excised compared with none (HR: $0.41 ; 95 \% \mathrm{CI}$ : $0.23-0.74$ ) and those who received either adjuvant
Table 3 Factors Associated with Receipt of Any Adjuvant Therapy (Radiotherapy, Chemotherapy or Multimodal)

\begin{tabular}{|c|c|c|c|c|}
\hline \multicolumn{2}{|l|}{ Characteristics: } & \multirow{2}{*}{$\begin{array}{c}\text { Adj. } \\
\text { OR }\end{array}$} & \multirow{2}{*}{$\begin{array}{l}95 \% \mathrm{Cl} \\
\text { ref }\end{array}$} & \multirow{2}{*}{$\begin{array}{l}p \text {-value } \\
-\end{array}$} \\
\hline Age at diagnosis & $<60$ & & & \\
\hline & $60-69$ & 0.25 & $0.05-1.30$ & 0.100 \\
\hline & $70-79$ & 0.10 & $0.02-0.62$ & 0.013 \\
\hline & $80+$ & 0.02 & $0.01-0.14$ & $<0.001$ \\
\hline \multirow{4}{*}{$\begin{array}{l}\text { Diagnostic } \\
\text { period }\end{array}$} & $1980-1989$ & 1.00 & ref & - \\
\hline & 1990-1999 & 0.14 & $0.02-0.68$ & 0.027 \\
\hline & $2000-2010$ & 0.23 & $0.03-1.20$ & 0.130 \\
\hline & $2010-2019$ & 0.87 & $0.09-4.74$ & 0.887 \\
\hline \multirow{2}{*}{$\begin{array}{l}\text { Place of } \\
\text { residence }\end{array}$} & Metropolitan & 1.00 & ref & - \\
\hline & $\begin{array}{l}\text { Non- } \\
\text { metropolitan }\end{array}$ & 0.55 & $0.15-1.99$ & 0.284 \\
\hline \multirow[t]{4}{*}{$\begin{array}{l}\text { Socioeconomic } \\
\text { (SEIFA) }\end{array}$} & $\begin{array}{l}\text { Most } \\
\text { disadvantaged }\end{array}$ & 1.00 & ref & - \\
\hline & Lower middle & 3.41 & $0.80-14.4$ & 0.096 \\
\hline & Upper middle & 1.31 & $0.32-5.45$ & 0.710 \\
\hline & $\begin{array}{l}\text { Least } \\
\text { disadvantaged }\end{array}$ & 1.47 & $0.37-5.89$ & $0.58 \mathrm{I}$ \\
\hline \multirow[t]{3}{*}{ Country of birth } & Australia & 1.00 & ref & - \\
\hline & $\begin{array}{l}\text { UK, Ireland, } \\
\text { NZ }\end{array}$ & 2.08 & $0.63-6.90$ & 0.228 \\
\hline & Other & 2.07 & $0.5 \mathrm{I}-8.47$ & 0.309 \\
\hline \multirow[t]{4}{*}{ FIGO stage } & I & 1.00 & ref & - \\
\hline & II & 1.92 & $0.24-15.3$ & 0.537 \\
\hline & III & 10.8 & $2.33-49.7$ & 0.002 \\
\hline & IV & 1.28 & $0.20-8.05$ & 0.793 \\
\hline \multirow{4}{*}{$\begin{array}{l}\text { Grade/ } \\
\text { differentiation: }\end{array}$} & Well & 1.00 & ref & - \\
\hline & Moderate & 3.57 & $0.09-89.9$ & 0.454 \\
\hline & $\begin{array}{l}\text { Poor/ } \\
\text { undifferentiated }\end{array}$ & 2.30 & $0.09-40.6$ & 0.568 \\
\hline & Unknown & 3.13 & $0.16-61.3$ & 0.452 \\
\hline \multirow{3}{*}{$\begin{array}{l}\text { Lymphovascular } \\
\text { invasion }\end{array}$} & No & 1.00 & ref & - \\
\hline & Yes & 0.73 & $0.19-2.82$ & 0.649 \\
\hline & Unknown & 0.25 & $0.05-1.40$ & 0.117 \\
\hline
\end{tabular}

(Continued) 
Table 3 (Continued).

\begin{tabular}{|l|l|l|l|l|}
\hline \multicolumn{2}{|l|}{ Characteristics: } & $\begin{array}{l}\text { Adj. } \\
\text { OR }\end{array}$ & $\mathbf{9 5 \% C l}$ & p-value \\
\hline \multirow{3}{*}{$\begin{array}{l}\text { Nodal } \\
\text { involvement }\end{array}$} & No & 1.00 & ref & - \\
\cline { 2 - 5 } & Yes & 0.89 & $0.13-5.96$ & 0.901 \\
\cline { 2 - 5 } & Unknown & 0.83 & $0.19-3.66$ & 0.807 \\
\hline \multirow{3}{*}{$\begin{array}{l}\text { Myometrial } \\
\text { involvement }\end{array}$} & No & 1.00 & ref & - \\
\cline { 2 - 5 } & Yes & 4.86 & $0.34-69.7$ & 0.245 \\
\cline { 2 - 5 } & Unknown & 2.45 & $0.07-82.5$ & 0.617 \\
\hline \multirow{3}{*}{$\begin{array}{l}\text { Classification } \\
\text { Homologous }\end{array}$} & 1.00 & ref & - \\
\cline { 2 - 5 } & Heterologous & 1.24 & $0.45-3.46$ & 0.699 \\
\cline { 2 - 5 } & Unknown & 2.49 & $0.05-48.9$ & 0.820 \\
\hline \multirow{2}{*}{$\begin{array}{l}\text { Sarcomatous } \\
\text { component }\end{array}$} & Minor & 1.00 & ref & - \\
\cline { 2 - 5 } & Major & 0.45 & $0.15-1.35$ & 0.156 \\
\cline { 2 - 5 } & Unknown & 1.13 & $0.04-34.4$ & 0.945 \\
\hline
\end{tabular}

Note: $n=139$, I case missing stage.

chemotherapy (HR: 0.39 ; 95\% CI: $0.18-0.84$ ) or multimodal therapy (HR: 0.11 ; 95\%CI: 0.06-0.30) compared with hysterectomy alone. No association was observed with respect to age or year of diagnosis. Analyses of DSM among those with in early stage disease, showed significant differences for FIGO stage II compares with stage I (HR: 5.01; 95\%CI: 1.71-14.6) and for multimodal therapy compared with hysterectomy alone (HR: 0.09; 95\%CI: 0.01-0.66). Nodal excision was not associated with statistically significant differences in DSM, however, point estimates were in the direction of reduced risk. For FIGO stage I alone, receipt of any adjuvant therapy modalities compared with surgery alone was associated with reduced DSM (HR: 0.31; 95\%CI: 0.10-0.96). For women with late stage disease (FIGO III/IV), nodal excision (HR: 0.29 ; 95\%CI: 0.14-0.61) and adjuvant treatment with chemotherapy (HR: 0.30 ; 95\%CI: $0.11-0.73$ ) or radio-chemotherapy (HR: 0.08 ; 95\%CI: $0.02-0.29$ ) were associated with reduced risk of uterine cancer death. Though not statistically significant, the direction of effect for adjuvant radiotherapy compared with hysterectomy alone was toward reduced risk for both early and late stage disease.
Results were similar for multivariable regression analyses of ACM, however no statistically significant differences were observed according to treatment approach or nodal excision for early stage disease (Supplementary Table 3). When considering the cohort overall, multimodal therapy was associated with reduced ACM compared with adjuvant chemotherapy alone (HR: $0.29 ; 95 \% \mathrm{CI}: 0.10-0.80$ )—results not shown.

\section{Discussion}

Our study describes the clinical characteristics, treatment patterns and outcomes for all locally resident cases of UCS managed within the main public teaching hospitals in South Australia over four decades. This moderately sized case series $(n=140)$ confirms the rare but aggressive nature of UCS and continuing need to improve early diagnosis and identify more effective treatments. Fifty percent of our cohort presented with late stage disease (FIGO III/IV) and 15\% with distant metastases at diagnosis. Furthermore, survival outcomes were poor, with a five-year disease specific survival of only $35 \%$ for the cohort overall. While use of adjuvant radiotherapy and multimodal chemo-radiotherapy increased over the study period, use of adjuvant chemotherapy alone declined in the 1990s with a gradual increase again thereafter. Despite changes in treatment approaches, no statistically significant improvement in survival was observed over time. Clinicopathological factors associated with reduced allcause mortality included earlier stage, adjuvant chemotherapy, multimodal therapy (vs surgery alone) and lymph node dissection. Adjuvant radiotherapy was not associated with improved survival outcomes. Similar findings were noted for the subgroup with late stage disease. However, none of the treatment factors were found be statistically significant in relation to all-cause mortality for early stage UCS, while only multimodal therapy was associated with reduced disease-specific mortality in this subgroup.

Disease characteristics of our case series were not dissimilar to those reported in other series, ${ }^{20-25}$ with approximately half presenting with extrauterine disease and $15 \%$ with distant metastases at diagnosis. The low proportion surviving to five years $(35 \%)$ is within previously reported ranges (30-39\%). ${ }^{26}$ We did not observe any statistically significant improvement in DSS or OS, which is consistent with Kanthan and Senger's assessment in their 2011 review that there had been no measurable improvement in UCS survival over the past four decades. ${ }^{15}$ 
Table 4 Disease Specific Survival

\begin{tabular}{|c|c|c|c|c|}
\hline \multirow[t]{2}{*}{ Factors: } & \multicolumn{4}{|c|}{$\%$ Survival ( $95 \%$ Confidence Intervals) at: } \\
\hline & I Year & 2 Years & 5 Years & P (Log Rank) \\
\hline All cases & $68(60-76)$ & $49(40-57)$ & $35(26-44)$ & \\
\hline \multicolumn{5}{|l|}{ Age at diagnosis (years) } \\
\hline$<60$ & $72(46-87)$ & $6 \mathrm{I}(35-79)$ & $55(30-74)$ & \\
\hline $60-69$ & $68(52-80)$ & $38(23-53)$ & $25(13-40)$ & $p=0.024$ \\
\hline $70-79$ & $76(61-80)$ & $60(44-73)$ & $44(29-60)$ & \\
\hline $80+$ & $53(33-71)$ & $36(18-55)$ & $18(6-36)$ & \\
\hline \multicolumn{5}{|l|}{ Diagnostic period: } \\
\hline $1980-1989$ & $66(43-81)$ & $28(12-48)$ & $14(4-32)$ & $p=0.183$ \\
\hline $1990-1999$ & $67(20-79)$ & $50(34-64)$ & $34(20-28)$ & \\
\hline $2000-2009$ & $63(44-77)$ & $50(32-66)$ & $37(21-54)$ & \\
\hline $2010-2019$ & $79(61-89)$ & $58(39-74)$ & $50(3 \mid-67)$ & \\
\hline \multicolumn{5}{|l|}{ Place of Residence: } \\
\hline Metropolitan & 71 (62-79) & $52(4 \mid-6 I)$ & $38(28-47)$ & $p=0.210$ \\
\hline Non-metropolitan & $59(39-75)$ & $37(20-55)$ & $25(10-42)$ & \\
\hline \multicolumn{5}{|l|}{ Socioeconomic (SEIFA): } \\
\hline Most disadvantaged & $72(55-83)$ & $42(27-56)$ & $34(20-49)$ & \\
\hline Lower middle & 77 (56-89) & $59(37-76)$ & $39(19-58)$ & $p=0.646$ \\
\hline Upper middle & $57(37-73)$ & $47(28-64)$ & $24(|0-4|)$ & \\
\hline Least disadvantaged & $67(49-8 I)$ & $49(32-65)$ & $43(26-59)$ & \\
\hline \multicolumn{5}{|l|}{ FIGO stage: } \\
\hline 1 & $83(7|-9|)$ & $68(54-79)$ & $52(37-65)$ & \\
\hline II & $58(23-82)$ & $35(8-64)$ & $23(4-53)$ & $p<0.001$ \\
\hline III & $73(58-85)$ & $45(30-59)$ & $31(18-45)$ & \\
\hline IV & $30(13-48)$ & $15(4-33)$ & 0 & \\
\hline \multicolumn{5}{|l|}{ Grade/differentiation: } \\
\hline Well & $60(13-88)$ & $60(13-88)$ & $60(13-88)$ & \\
\hline Moderate & $93(61-99)$ & $50(23-72)$ & $50(23-72)$ & $p=0.428$ \\
\hline Poor/undifferentiated & $64(53-73)$ & $48(37-58)$ & $32(22-43)$ & \\
\hline Uuknown & $72(5 \mid-87)$ & $48(26-58)$ & $30(13-49)$ & \\
\hline \multicolumn{5}{|l|}{ Lymphovascular invasion } \\
\hline No & $84(68-93)$ & $64(47-78)$ & $49(32-64)$ & \\
\hline Yes & $64(49-76)$ & $46(3 I-58)$ & $33(20-47)$ & $p=0.119$ \\
\hline Unknown & $61(45-74)$ & $38(24-53)$ & $25(13-39)$ & \\
\hline \multicolumn{5}{|l|}{ Heterologous } \\
\hline No & $68(56-78)$ & $47(35-58)$ & $37(25-48)$ & \\
\hline Yes & $72(56-85)$ & $62(46-75)$ & $39(25-54)$ & $p=0.192$ \\
\hline Unknown & $62(3 \mid-82)$ & $9(5-32)$ & $9(5-32)$ & \\
\hline \multicolumn{5}{|l|}{ Extent: } \\
\hline Myometrial involvement & $7 \mid(6 I-77)$ & $52(42-62$ & $36(26-45)$ & \\
\hline Omentum involvement & $4 \mid(2 \mid-60)$ & $26(10-45)$ & $21(7-40)$ & \\
\hline \multicolumn{5}{|l|}{ Lymph nodes removed } \\
\hline No & $40(20-59)$ & $30(12-50)$ & $20(6-39)$ & \\
\hline Yes & 79 (69-86) & $58(47-67)$ & $4 I(3 \mid-52)$ & $p=0.003$ \\
\hline Unknown & $47(22-69)$ & $16(3-39$ & $16(3-39)$ & \\
\hline
\end{tabular}

(Continued) 
Table 4 (Continued).

\begin{tabular}{|c|c|c|c|c|}
\hline \multirow[t]{2}{*}{ Factors: } & \multicolumn{4}{|c|}{$\%$ Survival (95\% Confidence Intervals) at: } \\
\hline & I Year & 2 Years & 5 Years & P (Log Rank) \\
\hline \multicolumn{5}{|l|}{ Lymph nodes positive } \\
\hline No & $87(73-94)$ & $68(52-80)$ & $52(36-66)$ & \\
\hline Yes & $88(60-97)$ & $59(33-78)$ & $369(16-62)$ & \\
\hline Unknown & $52(36-63)$ & $33(22-44)$ & $22(13-33)$ & \\
\hline \multicolumn{5}{|l|}{ Primary Treatment } \\
\hline Surgery only & $64(51-74)$ & $39(27-5 I)$ & $23(14-35)$ & \\
\hline Surgery + CT & $73(49-87)$ & $50(28-68)$ & $32(|4-5|)$ & $p<0.001$ \\
\hline Surgery + RT & $67(38-85)$ & $45(19-68)$ & $45(19-68)$ & \\
\hline Surgery + RT + CT & 95 (72-99) & $86(63-95)$ & 75 (49-89) & \\
\hline
\end{tabular}

Notes: Kaplan-Meier estimates of survival from UCS, according to sociodemographic and clinical characteristics $n=\mid 40$. Follow-up from date of diagnosis to date of death or last follow-up date.

Considerable uncertainty persists around the most effective treatment strategies for UCS. ${ }^{6,15,26-28}$ A total hysterectomy is the universally recommended primary treatment approach, and due to the high rate of recurrence

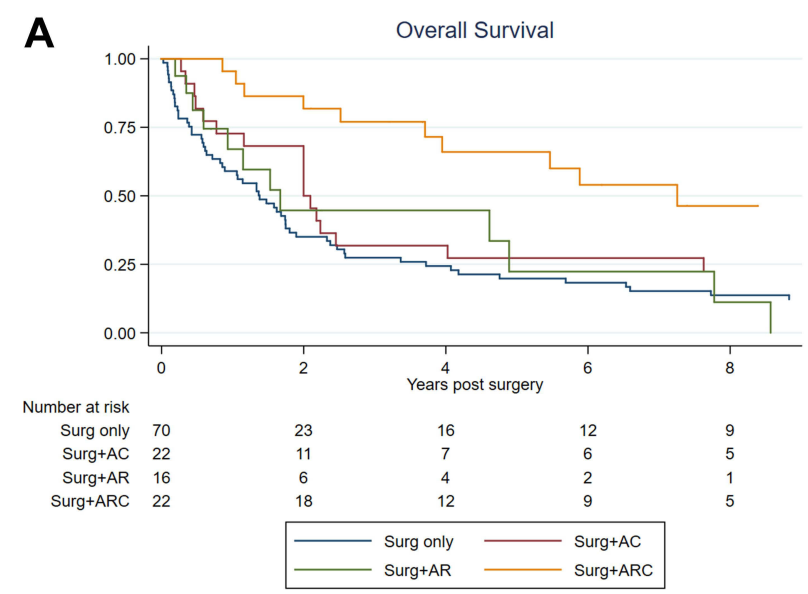

B

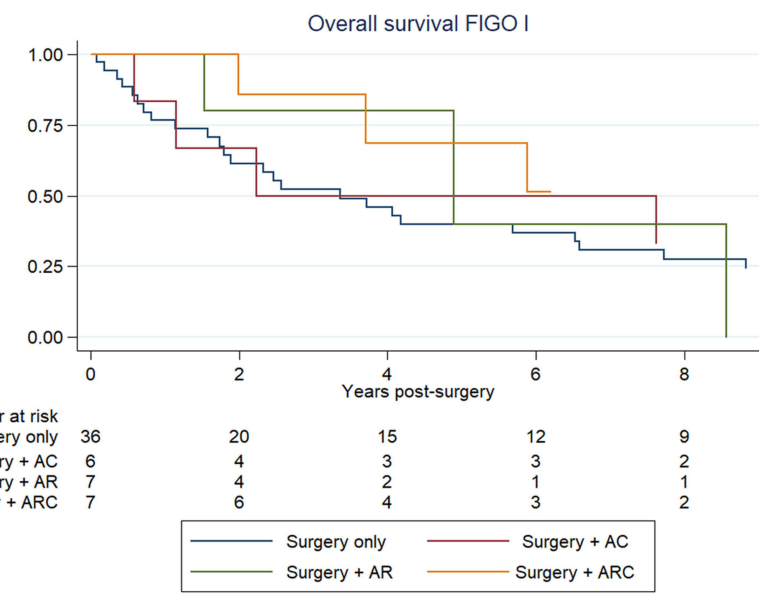

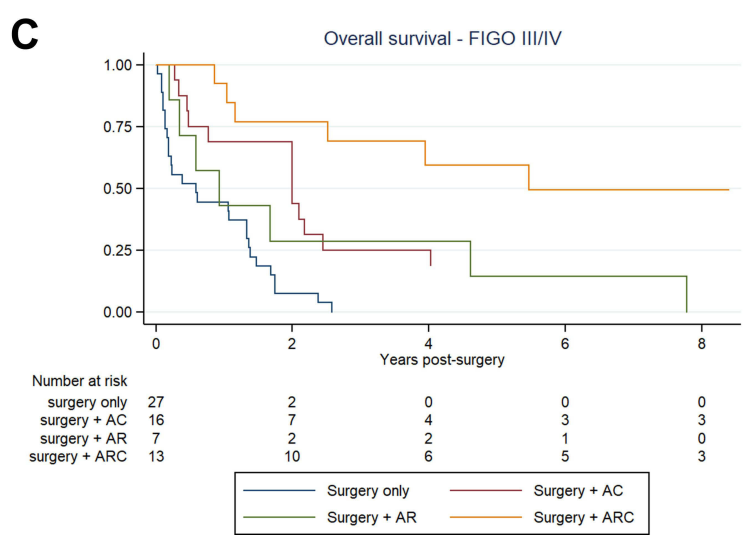

Figure I Overall survival among women with uterine carcinosarcomas according to primary treatment modality (SA, 1980-2016). (A) Whole cohort. (B) FIGO I (C) FIGO III/IV.

Abbreviations: Surg, surgery; AC, adjuvant chemotherapy; AR, adjuvant radiotherapy; ARC, adjuvant radio-chemotherapy (multimodal). 
Table 5 Disease Specific Mortality in Women Who Underwent Surgery ( \pm Adjuvant Therapies) for UCS

\begin{tabular}{|c|c|c|c|c|c|c|c|c|c|c|c|c|}
\hline \multirow[t]{2}{*}{ Characteristics: } & \multicolumn{3}{|c|}{ All Stages $(n=129)$} & \multicolumn{3}{|c|}{ FIGO I/II $(n=66)$} & \multicolumn{3}{|c|}{ FIGO III/IV (n=63) } & \multicolumn{3}{|c|}{ FIGO I $(n=56)$} \\
\hline & $\begin{array}{l}\text { Adj. } \\
\text { HR }\end{array}$ & $95 \% \mathrm{Cl}$ & $p$ & $\begin{array}{l}\text { Adj. } \\
\text { HR }\end{array}$ & $95 \% \mathrm{Cl}$ & $p$ & $\begin{array}{l}\text { Adj. } \\
\text { HR }\end{array}$ & $95 \% \mathrm{Cl}$ & $p$ & $\begin{array}{l}\text { Adj. } \\
\text { HR }\end{array}$ & $95 \% \mathrm{Cl}$ & $p$ \\
\hline Age at diagnosis & 0.99 & $0.96-1.01$ & 0.315 & 1.00 & $0.97-1.04$ & 0.969 & 0.97 & $0.93-1.01$ & 0.123 & 1.03 & $0.98-1.09$ & 0.263 \\
\hline Year of diagnosis & 1.01 & $0.98-1.04$ & 0.632 & 1.03 & $0.97-1.10$ & 0.355 & 1.00 & $0.96-1.04$ & 0.996 & 0.98 & $0.90-1.06$ & 0.565 \\
\hline \multicolumn{13}{|l|}{ FIGO stage: } \\
\hline I & 1.00 & ref & - & 1.00 & ref & - & - & - & - & na & - & - \\
\hline II & 4.46 & $1.89-10.5$ & 0.002 & 5.01 & $1.7|-| 4.6$ & 0.003 & - & - & - & - & - & - \\
\hline III & 3.00 & $1.57-5.76$ & 0.001 & - & - & - & 1.00 & ref & - & - & - & - \\
\hline IV & 7.24 & $3.7 I-14.2$ & $<0.001$ & - & - & - & 2.07 & $0.98-4.37$ & 0.056 & - & - & - \\
\hline \multicolumn{13}{|l|}{$\begin{array}{l}\text { Lymph nodes } \\
\text { removed }\end{array}$} \\
\hline No & 1.00 & ref & - & 1.00 & ref & & 1.00 & ref & - & 1.00 & - & - \\
\hline Yes & 0.41 & $0.23-0.74$ & 0.015 & 0.66 & $0.16-2.26$ & 0.553 & 0.29 & $0.14-0.61$ & 0.001 & $0.4 I$ & $0.07-2.26$ & 0.305 \\
\hline \multicolumn{13}{|l|}{ Treatments: } \\
\hline Surgery only & 1.00 & ref & - & 1.00 & ref & - & 1.00 & ref & - & 1.00 & - & - \\
\hline Surgery + AC & 0.39 & $0.18-0.84$ & 0.016 & 1.09 & $0.28-4.36$ & 0.896 & 0.30 & $0.11-0.73$ & 0.010 & 1.04 & $0.25-4.32$ & 0.952 \\
\hline Surgery + AR & 0.56 & $0.27-1.62$ & 0.170 & 0.41 & $0.09-1.77$ & 0.232 & 0.50 & $0.16-1.54$ & 0.226 & 0.27 & $0.03-2.62$ & 0.257 \\
\hline Surgery + ARC & 0.11 & $0.06-0.30$ & $<0.001$ & 0.09 & $0.01-0.66$ & 0.017 & 0.08 & $0.02-0.29$ & $<0.001$ & 0.20 & $0.02-1.82$ & 0.153 \\
\hline $\begin{array}{l}\text { (Any adjuvant } \\
\text { therapy) }\end{array}$ & 0.31 & $0.16-0.60$ & $<0.001$ & 0.45 & $0.17-1.22$ & 0.117 & 0.29 & $0.16-0.53$ & $<0.001$ & 0.31 & $0.10-0.96$ & 0.043 \\
\hline
\end{tabular}

Notes: Multivariable Cox regression for risk of uterine cancer death with age and diagnosis year as continuous variables. Follow-up time=date of diagnosis to date of death or last follow-up date. Sociodemographic and clinical variables with no association included socioeconomic status, place of residence, country of birth, grade, I-VI lymphovascular invasion, myometrial involvement and heterologous cell types-these variables were not included in the final models.

Abbreviations: AC, adjuvant chemotherapy; AR, adjuvant radiotherapy; ARC, adjuvant radiotherapy and chemotherapy.

of UCS $(50-80 \%){ }^{15}$ adjuvant therapy has also been recommended. $^{26}$ While the majority of our cohort had surgery, adjuvant therapies were used less universally (44\% overall had received some form of adjuvant therapy), with age, diagnostic period, and stage being the most influential determinants. Older age was associated with decreased likelihood of receiving any adjuvant therapy, as has been reported previously. ${ }^{29}$ Chemotherapy was more likely to be provided to women with advanced disease (most frequently FIGO III), and those with a heterologous sarcomatous component, while receipt of adjuvant radiotherapy was not associated with stage of disease, or any other clinical characteristics. Adjuvant radiotherapy and combined multimodal therapy, however, have increased in use over more recent periods.

While we found that adjuvant chemotherapy alone was associated with reduced risk of all-cause mortality for the whole cohort and for FIGO stage III/IV, no association was found among those who presented with FIGO Stage I-II or for Stage I alone. This may be due to lack of power, particularly in subgroup analyses. Though others have reported improved survival outcomes with adjuvant chemotherapy in subgroups analyses for early stage UCS, ${ }^{21}$ most smaller series have not found any statistically significant improvement. ${ }^{22,29-31}$

Our findings of no association between receipt of adjuvant radiotherapy and disease specific or overall survival are consistent with a number of other relatively small retrospective studies ${ }^{21,23,29,31,32}$ and two previous randomized trials ${ }^{33,34}$ that showed reduced locoregional recurrence but no independent effect on survival. Conversely, in larger studies utilizing SEER data, Manzerova et $\mathrm{al}^{35}$ and $\mathrm{Li}$ et $\mathrm{al}^{24}$ have reported a statistically significant improvement in overall survival among those who received radiotherapy, with risk reductions in the order of $25 \%$. However, no adjustment was 
made for receipt of adjuvant chemotherapy in these studies. In an analysis of population-wide registry data from The Netherlands, where receipt of chemotherapy was taken into account, Versluis et $\mathrm{al}^{7}$ reported improved overall survival among patients who received adjuvant RT for the whole study population, though no stagespecific analyses were reported. Further investigation of the role of radiotherapy is required, preferably within a randomized controlled trial.

While evidence is still quite limited, results from several retrospective studies have indicated improved outcomes with multimodal therapies incorporating postsurgical chemotherapy and radiotherapy., ${ }^{76,27}$ However, other case series have not identified any survival benefit. ${ }^{21,22,25,30,31,36}$ In our study, multimodal therapy was associated with reduced risk of allcause mortality for both early and late stage disease. This is in contrast with the lack of association reported in previous studies that have assessed multimodal therapy specifically in early stage disease. ${ }^{16,21,25,30,31}$ Insufficient power may explain the null findings in most of these cases.

We also observed a survival benefit with respect to lymph node dissection, as have others. ${ }^{24,32,37}$ However, some previous studies have reported a threshold regarding number of excised nodes required to impact patient survival (eg, $>10$ nodes $^{7}$ and $>11^{8}$ ) while other relatively small studies have found no association. ${ }^{38,39}$ Our analyses found statistically significant reductions in mortality with pelvic lymph node dissection with a median number of six excised nodes (interquartile range 2-12). Though suggestive of a survival benefit in early stage disease, our findings for FIGO stage I-II, and stage I alone, were not statistically significant. Further research is required to provide more definitive conclusions in early stage UCS.

Cautious interpretation of our findings is warranted, particularly in relation to differences in survival outcomes according to treatment modality, due to probable biases inherent in observational studies. Selection of adjuvant therapy modalities is likely to be influenced by women's general health and other confounding factors, which we were not able to account for in multivariable models. Furthermore, there have been heterogeneous and changing treatment practices over the study period.
Our study also lacked statistical power due to the small number of cases/rarity of UCS. However, careful review of pathologic reports has ensured only verified cases of UCS were included. Since data on patterns and dates of recurrence were incomplete for our cohort, we were unable to determine factors associated with risk of locoregional recurrence or distant metastasis. While our patient series represents women treated within public teaching hospitals in Adelaide, it is likely to account for a majority of cases in South Australia, given the centralized tertiary health-care system and geography of the state's population.

\section{Conclusion}

Our findings indicate that multimodal adjuvant therapy after hysterectomy for UCS is associated with better survival outcomes. However, prognosis for UCS remains very poor and has not improved significantly in the past decades despite advances in adjuvant therapies and trend toward multimodal treatment protocols. The development of more effective targeted treatments is, therefore, urgently needed to improve the outcome for women with UCS.

\section{Abbreviations}

ACM, all-cause mortality; CI, confidence interval; DSM, disease specific mortality; DSS, disease specific survival; EBRT, external beam radiotherapy; FIGO, International Federation of Gynecology and Obstetrics; HR, hazard ratio; OR, odds ratio; OS, overall survival; SACCR, South Australian Clinical Cancer Registry; SEIFA, Socioeconomic Indexes for Area; UCS, uterine carcinosarcoma.

\section{Data Sharing Statement}

Data used in this study are not publicly available and can only be accessed, with appropriate approvals from data custodians and ethical clearance, from Wellbeing SA (Health dept of South Australia).

\section{Ethics Approval and Informed Consent}

Ethical approval for this study was granted by the SA Department for Health and Ageing Human Research Ethics Committee Reference: HREC/17/SAH/69 with 
a waiver of consent for use of deidentified data from the clinical registry.

\section{Funding}

Kerri Beckmann is supported through a National Health and Medical Research Council Early Career Researcher Fellowship.

\section{Disclosure}

The authors report no conflicts of interest in this work.

\section{References}

1. Matsuo K, Ross MS, Machida H, Blake EA, Roman LD. Trends of uterine carcinosarcoma in the United States. $J$ Gynecol Oncol. 2018;29(2):e22. doi:10.3802/jgo.2018.29.e22

2. Denschlag D, Ulrich UA. Uterine Carcinosarcomas - Diagnosis and Management. Oncol Res Treat. 2018;41(11):675-679. doi:10.1159/ 000494335

3. Kernochan LE, Garcia RL. Carcinosarcomas (malignant mixed Mullerian tumor) of the uterus: advances in elucidation of biologic and clinical characteristics. J Natl Compr Canc Netw. 2009;7(5):550-556; quiz 557. doi:10.6004/jncen.2009.0037

4. Zelmanowicz A, Hildesheim A, Sherman ME, et al. Evidence for a common etiology for endometrial carcinomas and malignant mixed mullerian tumors. Gynecol Oncol. 1998;69(3):253-257. doi:10.1006/ gyno.1998.4941

5. Cantrell LA, Blank SV, Duska LR. Uterine carcinosarcoma: a review of the literature. Gynecol Oncol. 2015;137(3):581-588. doi:10.1016/ j.ygyno.2015.03.041

6. Vorgias G, Fotiou S. The role of lymphadenectomy in uterine carcinosarcomas (malignant mixed mullerian tumours): a critical literature review. Arch Gynecol Obstet. 2010;282(6):659-664. doi:10.1007/ s00404-010-1649-0

7. Versluis MAC, Pielsticker C, van der Aa MA, de Bruyn M, Hollema H, Nijman HW. Lymphadenectomy and adjuvant therapy improve survival with uterine carcinosarcoma: a large retrospective cohort Study. Oncology. 2018;95(2):100-108. doi:10.1159/000488531

8. Temkin SM, Hellmann M, Lee YC, Abulafia O. Early-stage carcinosarcoma of the uterus: the significance of lymph node count. Int J Gynecol Cancer. 2007;17(1):215-219. doi:10.1111/j.15251438.2006.00762.x

9. Menczer J. Review of recommended treatment of uterine carcinosarcoma. Curr Treat Options Oncol. 2015;16(11):53. doi:10.1007/s11864-015-0370-4

10. Homesley HD, Filiaci V, Markman M, et al. Phase III trial of ifosfamide with or without paclitaxel in advanced uterine carcinosarcoma: a Gynecologic Oncology Group Study. J Clin Oncol. 2007;25 (5):526-531. doi:10.1200/JCO.2006.06.4907

11. Sutton G, Kauderer J, Carson LF, et al. Adjuvant ifosfamide and cisplatin in patients with completely resected stage I or II carcinosarcomas (mixed mesodermal tumors) of the uterus: a Gynecologic Oncology Group study. Gynecol Oncol. 2005;96(3):630-634. doi:10.1016/j.ygyno.2004.11.022

12. Heinzelmann-Schwarz V, Kind AB, Vetter M, et al. Should MMMT still be treated with adjuvant taxane-based combination chemotherapy? J Cancer Res Clin Oncol. 2020;146(3):695-704. doi:10.1007/s00432-019-03091-y
13. Powell MA, Filiaci VL, Rose PG, et al. Phase II evaluation of paclitaxel and carboplatin in the treatment of carcinosarcoma of the uterus: a Gynecologic Oncology Group study. J Clin Oncol. 2010;28 (16):2727-2731. doi:10.1200/JCO.2009.26.8326

14. Berton-Rigaud D, Devouassoux-Shisheboran M, Ledermann JA, et al. Gynecologic cancer interGroup (GCIG) consensus review for uterine and ovarian carcinosarcoma. Int J Gynecol Cancer. 2014;24(9 Suppl 3):S55-60. doi:10.1097/IGC.0000000000000228

15. Kanthan R, Senger JL. Uterine carcinosarcomas (malignant mixed mullerian tumours): a review with special emphasis on the controversies in management. Obstet Gynecol Int. 2011;2011:470795. doi: $10.1155 / 2011 / 470795$

16. Gonzalez Bosquet J, Terstriep SA, Cliby WA, et al. The impact of multi-modal therapy on survival for uterine carcinosarcomas. Gynecol Oncol. 2010;116(3):419-423. doi:10.1016/j.ygyno.2009.10.053

17. Australian Bureau of Statisitics. SEIFA 2011 socio-economic indexes for areas; 2011.

18. Department of Health and Aged Care. Measuring remoteness: Accessibility/Remoteness Index of Australia (ARIA), Revised Edition. Occasional Paper. Canberra: Department of Health and Aged Care 2011.

19. National Comprehensive Cancer Network. NCCN clinical practice guidelines in oncology: uterine neoplasms v1.2021. NCCN.org; 2020. Accessed February 16, 2021.

20. Abdulfatah E, Lordello L, Khurram M, et al. Predictive histologic factors in carcinosarcomas of the uterus: a multi-institutional Study. Int J Gynecol Pathol. 2019;38(3):205-215. doi:10.1097/PGP.0000000000000497

21. Dickson EL, Vogel RI, Gehrig PA, et al. A multi-institutional study of outcomes in stage I-III uterine carcinosarcoma. Gynecol Oncol. 2015;139(2):275-282. doi:10.1016/j.ygyno.2015.09.002

22. Makker V, Abu-Rustum NR, Alektiar KM, et al. A retrospective assessment of outcomes of chemotherapy-based versus radiation-only adjuvant treatment for completely resected stage I-IV uterine carcinosarcoma. Gynecol Oncol. 2008;111(2):249-254. doi:10.1016/j.ygyno.2008.06.035

23. Cha J, Kim YS, Park W, et al. Clinical significance of radiotherapy in patients with primary uterine carcinosarcoma: a multicenter retrospective study (KROG 13-08). J Gynecol Oncol. 2016;27(6):e58. doi:10.3802/jgo.2016.27.e58

24. Li Y, Ren H, Wang J. Outcome of adjuvant radiotherapy after total hysterectomy in patients with uterine leiomyosarcoma or carcinosarcoma: a SEER-based study. BMC Cancer. 2019;19(1):697. doi:10.1186/s12885-019-5879-7

25. Gungorduk K, Ozdemir A, Ertas IE, et al. Adjuvant treatment modalities, prognostic predictors and outcomes of uterine carcinosarcomas. Cancer Res Treat. 2015;47(2):282-289. doi:10.4143/crt.2014.009

26. Vitale SG, Lagana AS, Capriglione S, et al. Target therapies for uterine carcinosarcomas: current evidence and future perspectives. Int J Mol Sci. 2017;18(5):1100. doi:10.3390/ijms 18051100

27. Menczer J, Levy T, Piura B, et al. A comparison between different postoperative treatment modalities of uterine carcinosarcoma. Gynecol Oncol. 2005;97(1):166-170. doi:10.1016/j. ygyno.2004.12.006

28. Matsuzaki S, Klar M, Matsuzaki S, Roman LD, Sood AK, Matsuo K. Uterine carcinosarcoma: contemporary clinical summary, molecular updates, and future research opportunity. Gynecol Oncol. 2020.

29. Garg G, Yee C, Schwartz K, Mutch DG, Morris RT, Powell MA. Patterns of care, predictors, and outcomes of chemotherapy in elderly women with early-stage uterine carcinosarcoma: a population-based analysis. Gynecol Oncol. 2014;133(2):242-249. doi:10.1016/j. ygyno.2014.02.021 
30. Kimyon Comert G, Turkmen O, Boyraz G, et al. Effect of Adjuvant Therapy on Oncologic Outcomes of Surgically Confirmed Stage I Uterine Carcinosarcoma: a Turkish Gynecologic Oncology Study. Balkan Med J. 2019;36(4):229-234. doi:10.4274/balkanmedj. galenos.2019.2018.12.75

31. Kurnit KC, Previs RA, Soliman PT, et al. Prognostic factors impacting survival in early stage uterine carcinosarcoma. Gynecol Oncol. 2019;152(1):31-37. doi:10.1016/j.ygyno.2018.10.034

32. Nemani D, Mitra N, Guo M, Lin L. Assessing the effects of lymphadenectomy and radiation therapy in patients with uterine carcinosarcoma: a SEER analysis. Gynecol Oncol. 2008;111(1):82-88. doi:10.1016/j.ygyno.2008.05.016

33. Reed NS, Mangioni C, Malmstrom H, et al. Phase III randomised study to evaluate the role of adjuvant pelvic radiotherapy in the treatment of uterine sarcomas stages I and II: an European Organisation for Research and Treatment of Cancer Gynaecological Cancer Group Study (protocol 55874). Eur J Cancer. 2008;44 (6):808-818. doi:10.1016/j.ejca.2008.01.019

34. Wolfson AH, Brady MF, Rocereto T, et al. A gynecologic oncology group randomized phase III trial of whole abdominal irradiation (WAI) vs. cisplatin-ifosfamide and mesna (CIM) as post-surgical therapy in stage I-IV carcinosarcoma (CS) of the uterus. Gynecol Oncol. 2007;107(2):177-185. doi:10.1016/j.ygyno.2007.07.070
35. Manzerova J, Sison CP, Gupta D, et al. Adjuvant radiation therapy in uterine carcinosarcoma: a population-based analysis of patient demographic and clinical characteristics, patterns of care and outcomes. Gynecol Oncol. 2016;141(2):225-230. doi:10.1016/j.ygyno. 2016.02 .013

36. Zhu J, Wen $\mathrm{H}$, Bi R, Wu X. Clinicopathological characteristics, treatment and outcomes in uterine carcinosarcoma and grade 3 endometrial cancer patients: a comparative study. J Gynecol Oncol. 2016;27(2):e18. doi:10.3802/jgo.2016.27.e18

37. Alagkiozidis I, Weedon J, Grossman A, et al. Extent of lymph node dissection and overall survival in patients with uterine carcinosarcoma, papillary serous and endometrioid adenocarcinoma: a retrospective cohort study. Int J Surg. 2015;24(Pt A):9-13. doi:10.1016/j.ijsu.2015.10.006

38. Otsuki A, Otsuki T, Tokunaga H, et al. Evaluation of postoperative chemotherapy in patients with uterine carcinosarcoma: a retrospective survey of the Tohoku Gynecologic Cancer Unit Int J Clin Oncol. 2015;20(3):574-578. doi:10.1007/s10147-0140732-0

39. Sagae S, Yamashita K, Ishioka S, et al. Preoperative diagnosis and treatment results in 106 patients with uterine sarcoma in Hokkaido, Japan. Oncology. 2004;67(1):33-39. doi:10.1159/000080283

\section{Publish your work in this journal}

Cancer Management and Research is an international, peer-reviewed open access journal focusing on cancer research and the optimal use of preventative and integrated treatment interventions to achieve improved outcomes, enhanced survival and quality of life for the cancer patient.
The manuscript management system is completely online and includes a very quick and fair peer-review system, which is all easy to use. Visit http://www.dovepress.com/testimonials.php to read real quotes from published authors. 